\title{
HELIOSPHERIC EFFECTS ON COSMIC-RAY ELECTRONS ${ }^{1}$
}

\author{
H. MoraAl, ${ }^{2}$ J. R. Jokipir, ${ }^{3}$ AND R. A. MewaldT ${ }^{4}$ \\ Received 1990 April 25 ; accepted 1990 June 15
}

\begin{abstract}
Motivated by the success of current modulation models in interpreting the solar modulation of Galactic cosmic-ray nuclei, we consider several effects on cosmic-ray electrons which are new or which have not yet been fully explored. Among these are the contribution of Galactic positrons to the total electron flux, the production of secondary electrons from solar system objects, and the possibility of electrons being accelerated at the solar wind termination shock ("anomalous" cosmic-ray electrons). We find that the first of these provides a natural interpretation of the time variations of Galactic cosmic-ray electrons. Jovian electrons and thermal solar wind electrons may be accelerated to cosmic-ray energies at the termination shock. Such acceleration effects may alter the time-intensity profiles of electrons at Earth, further modifying the interpretation of the electron modulation.
\end{abstract}

Subject headings: cosmic rays: general - particle acceleration

\section{INTRODUCTION}

Over the past several years it has become apparent that the well-known 11 yr cosmic-ray solar modulation cycle is actually a 22 yr cycle, driven by the 22 yr cycle for the reversal of the solar magnetic field. The 22 yr nature of the solar modulation cycle is believed to be due principally to gradient and curvature drifts in the large-scale heliospheric magnetic field that change direction every $11 \mathrm{yr}$ as the direction of the field reverses (Jokipii and Thomas 1981; Kóta and Jokipii 1983). Solar modulation models that take into account particle drifts within a geometry that employs a wavy neutral current sheet are successful in explaining both the well-known $11 \mathrm{yr}$ and 22 yr cosmic-ray intensity variations. Two of the most significant observed features are the following:

1. In the 1965 and 1987 solar minima the cosmic-ray intensities show a sharp peak at maximum, while in the intervening 1975 solar minimum the intensity exhibited a flat maximum which lasted for several years. Jokipii and Thomas (1981) and Kóta and Jokipii (1983) showed that this phenomenon is naturally explained by drift effects in connection with the change in sign of the heliospheric magnetic field between successive sunspot minima.

2. Measurements of anomalous cosmic rays (e.g., Cummings, Stoke, and Webber 1990; Cummings et al. 1990; McKibben 1989) show that the latitudinal gradient of cosmic rays near the ecliptic plane was positive around the 1976 solar minimum but negative around the next minimum in 1987. This is a fundamental prediction of drift models (e.g., Jokipii and Kopriva 1979). Moraal $(1990 a, b)$ found that the predictions of a drift model are in general agreement with these observations.

These observations and others carried out over three solar cycles, together with their natural interpretation in terms of the change in the sign of the solar magnetic field, support strongly the existence of a 22 yr cycle in cosmic-ray modulation caused by the 22 yr solar magnetic cycle. Up to now, modeling efforts

\footnotetext{
${ }^{1}$ Contribution No. $90-15$ of the University of Arizona Theoretical Astrophysics Program

${ }^{2}$ California Institute of Technology; permanently at Potchefstroom University, South Africa.

${ }^{3}$ University of Arizona, Tucson, AZ.

${ }^{4}$ California Institute of Technology.
}

have almost exclusively concentrated on positively charged cosmic-ray nuclei because observations of this component are most complete. Electrons, being negatively charged, should experience the phenomena mentioned above $11 \mathrm{yr}$ out of phase with the nuclei. Measurements of cosmic-ray electron density gradients do not exist, but Garcia-Munoz et al. $(1986,1987)$ have shown that the time variations of electron intensities are not as predicted by drift models which consider only negatrons. In addition, during solar maximum periods, when the heliospheric magnetic field reverses polarity, drift models predict a large excursion in the ratio of negatron to nuclei intensities. Such excursions are in fact observed, but they seem to be nearly an order of magnitude smaller than predicted by drift models.

We find below that including a finite fraction of Galactic positrons with negatrons in our models resolves many of the apparent discrepancies with the electron observations. Similar calculations in this regard were done by Potgieter and Burger (1990), but the present calculations are for a magnetic field model that deviates strongly from the Parker spiral structure above the solar poles. Such a model permits much more realistic diffusion coefficients.

This model explains many (but perhaps not all) of the details of the electron modulation in one conceptual framework. Its success also leads us to reinvestigate such questions as other possible source(s) of electrons $(\S \mathrm{VI})$, and their acceleration mechanism(s) (§ VII). These sections are intended to show that previously neglected source or acceleration mechanisms or both may have further profound influences on our understanding of the modulation of cosmic-ray electrons.

\section{THE POSITRON CONTENT IN THE COSMIC-RAY} ELECTRON FLUX

Cosmic-ray electrons are observed to consist of both negatrons and positrons. The positron fraction of the intensity $(j)$ is defined as

$$
f_{+}=\frac{j\left(e^{+}\right)}{j\left(e^{-}\right)+j\left(e^{+}\right)} .
$$

The principal sources of secondary electrons in the Galaxy are the $\pi^{-} \rightarrow \mu^{-} \rightarrow e^{-}$and $\pi^{+} \rightarrow \mu^{+} \rightarrow e^{+}$decay chains, which 


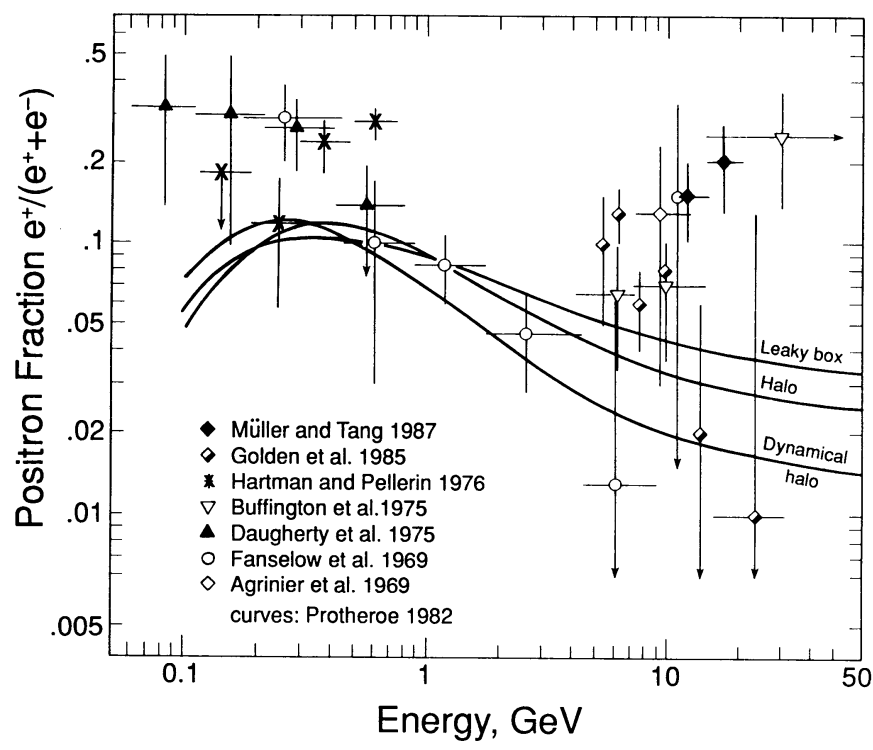

FIG. 1.- Observed and calculated fraction of positrons in the total electron flux. Observations were all made near Earth, while calculations are for the interstellar fraction. Neither is corrected for modulation. Key to references: Müller and Tang (1987); $\diamond$, Golden et al. (1985); *, Hartman and Pellerin (1976); $\nabla$, Buffington et al. (1975); $\Delta$, Daugherty et al. (1985); $\bigcirc$, Fanselow et al. (1969); $\diamond$, Agrinier et al. (1969); curves, Protheroe (1982).

result in roughly equal numbers of negatrons $\left(e^{-}\right)$and positrons $\left(e^{+}\right)$. Figure 1 compiles most of the existing measurements of the positron fraction above $50 \mathrm{MeV}$, together with three curves calculated by Protheroe (1982) for three models of Galactic propagation. The observed values of $f_{+}$vary from $\approx 1 \%$ to $30 \%$. The scatter of the data points and the large error bars reflect the difficulty of the measurement (see the references in Fig. 1). It should be pointed out that because these observations were all made by balloon-borne instruments, they require sizable corrections for the flux of "secondary" positrons and negatrons, produced in the atmosphere by interactions of cosmic-ray nuclei. As a result there may be systematic errors in the measurements, particularly at the lowest energies, where as much as $50 \%$ of the observed positron flux may be atmospheric secondaries (see, e.g., Fanselow et al. 1969). For the purpose of modulation studies, authors have traditionallyand without justification - assumed that the positron fraction is small, i.e., $\lesssim 10 \%$, as predicted by the propagation models shown in this figure. If this assumption were valid, the total electron spectrum would be expected to undergo modulation as if it consisted mostly of negatrons, and the positron contribution could be safely ignored. Figure 1 clearly shows, however, that at kinetic energies $T \lesssim 1 \mathrm{GeV}$, most of the measurements lie well above $f_{+}=0.1$, and the average may be closer to $f_{+}=0.2$.

In the next section we demonstrate that drift effects in the heliosphere are quite likely to contribute to this high positron content observed at Earth below about $1 \mathrm{GeV}$, and that these drifts will also strongly modify the temporal variation of the total electron intensity.

\section{SIMULTANEOUS MODULATION OF COSMIC-RAY} NEGATRONS AND POSITRONS

Cosmic-ray particles are subjected to four distinct transport effects which contribute to two distinct kinds of motion.
Regular motion occurs because the particles are convected with the plasma and magnetic field and because they drift in the large-scale magnetic field. At the same time there is a random walk of the particles caused by scattering off random magnetic irregularities. Because the random walk normal to the field is less rapid, the diffusion tensor is anisotropic. It may be written in terms of the perpendicular coefficient $\kappa_{\perp}$, the parallel coefficient $\kappa_{\|}$, and the antisymmetric, or drift coefficient $\kappa_{T}$, and the components of the local magnetic field $\boldsymbol{B}$ as

$$
\kappa_{i j}=\kappa_{\perp} \delta_{i j}-\frac{\left(\kappa_{\perp}-\kappa_{\|}\right) B_{i} B_{j}}{B^{2}}+\frac{\epsilon_{i j k} \kappa_{T} B_{k}}{B} .
$$

Because of the $\boldsymbol{V} \times \boldsymbol{B}$ electric field in the wind, there are associated energy changes.

The resulting transport is a superposition of these coherent and random effects. They were combined first by Parker (1965) to obtain the generally accepted transport equation for the quasi-isotropic distribution function $f(r, p, t)$ of cosmic rays of momentum $p$ at position $r$ and time $t$ :

$$
\begin{array}{rlrl}
\frac{\partial f}{\partial t}= & \frac{\partial}{\partial x_{i}}\left(\kappa_{i j}^{s} \frac{\partial f}{\partial x_{j}}\right) & & \text { (diffusion) }, \\
& -U_{i} \frac{\partial f}{\partial x_{i}} & & \text { (convection), } \\
& -V_{d i} \frac{\partial f}{\partial x_{i}} & & \text { (guiding-center drift) } \\
& +\frac{1}{3} \frac{\partial U_{i}}{\partial x_{i}}\left(\frac{\partial f}{\partial \ln p}\right) & & \text { (energy change) }, \\
& +Q\left(x_{i}, t, p\right) & \text { (source), }
\end{array}
$$

where $\kappa_{i j}^{S}$ is the symmetric part of $\kappa_{i j}$. The labels next to each of the various terms indicate the associated physical effect. The guiding-center drift velocity is the divergence of the antisymmetric part of $\kappa_{i j}$ and may be given in terms of the local magnetic field $\boldsymbol{B}$ and the particle charge $q$ by $\boldsymbol{V}_{d}=(p w / 3 q)$ $\nabla \times\left(B / B^{2}\right)$ for the case where the scattering mean free path is significantly larger than the gyroradius (with $B$ in SI units).

In each hemisphere the field is generally assumed to be a classical Parker Archimedean spiral, with the sense of the field being outward in one hemisphere and inward in the other. At sunspot minimum, the current sheet is nearly equatorial. The field direction alternates with each $11 \mathrm{yr}$ sunspot cycle, so that during the 1976 sunspot minimum, the northern field was directed outward from the Sun, but in 1965 and 1987 the northern field pointed inward. Observations indicate that the inclination of the current sheet increases as a function of time toward sunspot maximum, but the structure for the years near sunspot maximum is not simple. In the rest of the discussion, it will be generally assumed that the overall magnetic structure is given by this model, recognizing that it may not be a particularly good representation in the few years around maximum sunspot activity.

We present modulated electron intensities, calculated from a simple steady state two-dimensional (radial distance, polar angle) modulation model. In the calculations discussed here, the transport equation (2) was solved numerically over 73 grid points from North to South Pole. At the two poles the intensities were assumed to be symmetric. In radial distance there were 101 grid points, with an interstellar spectrum, taken from Protheroe (1982), imposed on the outer boundary, which was 
specified at a radial distance of $60 \mathrm{AU}$. The Sun was assumed to be a reflector of cosmic rays, and the initial no modulation condition was set at a rigidity of $20 \mathrm{GV}$.

This model has an actual wavy neutral sheet in it, where the direction of the magnetic field spirals abruptly switch direction. Because of the underlying axisymmetry of a two-dimensional model, such a wavy sheet violates the divergence-free character of the magnetic field. The only validity for simulating the actual, three-dimensional nature of the sheet in this way is that the solutions can be calibrated against and agree well with the full three-dimensional results of Kóta and Jokipii (1983). The diffusion coefficients used were identical to those in Moraal $(1990 a)$ and are of the form

$$
\kappa_{\|}=2.4 \times 10^{22} \beta\left(P / P_{0}\right)\left|B_{e} / B\right| \mathrm{cm}^{2} \mathrm{~s}^{-1},
$$

and

$$
\kappa_{\perp}=2.2 \times 10^{20} \beta f(P)\left|B_{e} / B\right| \mathrm{cm}^{2} \mathrm{~s}^{-1},
$$

where $P$ is rigidity in $\mathrm{GV}, P_{0}=1 \mathrm{GV}, \beta$ is the particle speed relative to the speed of light, and $B$ is the magnetic field strength, with the subscript $e$ referring to its value at Earth.The function $f(P)=\sqrt{P / P_{0}}$ if $P>P_{0}$, and $f(P)=1$ if $P \leq P_{0}$. We took $P_{0}=1 \mathrm{GV}$. The drift coefficient (in SI units) is $\kappa_{T}=$ $\beta P /(3 B)$, and the magnitude of the drift velocity value of $B_{e}$ was set by selecting $B_{e}=8 \mathrm{nT}$. This field takes account of the inhibiting effect of long-wavelength turbulence on drift velocities, as calculated by Burger and Moraal (1990).

Jokipii and Kóta (1989) suggested that the radial field lines above the solar poles, as predicted by the Parker spiral structure, might in fact be considerably modified because of supergranulation effects at the solar surface. They also stated that this would have a significant effect on cosmic-ray modulation. This may lead to a strongly enhanced average scalar field magnitude at large radial distances above the solar poles. This effect was crudely modeled by multiplying the Parker spiral by a hyperbolic cosine in polar angle, $\theta$. such that it has the value one at $\theta=90^{\circ}$, two at $\theta=30^{\circ}, 150^{\circ}$, and 10 at the solar poles $(\theta=0)$.

It was shown by Moraal (1990a) that use of these parameters in this model leads to computed proton intensities, in good agreement with proton modulation observed at Earth and on the Pioneer/Voyager spacecraft.Without this field modification there is no set of reasonable diffusion coefficients that can match the observations. This may serve as an indication that the modified field model indeed is correct.

Figure 2 shows the calculated electron spectra at Earth for these parameters, for both the $A>0$ and $A<0$ magnetic cycles. These two cases designate the states where the solar magnetic dipole moment is aligned with the rotation axis (as in the approximate $11 \mathrm{yr}$ period 1970 to 1980 ) and oppositely directed to it (as in the $11 \mathrm{yr}$ periods adjacent to this interval), respectively. These computed spectra are in good agreement with observations.

The five solutions with $A<0$ are shown by solid lines, and from top to bottom are for neutral sheet tilt angles $\alpha=0^{\circ}, 10^{\circ}$, $20^{\circ}, 40^{\circ}, 70^{\circ}$, respectively. For $A>0$ we only show the $\alpha=0^{\circ}$ and $\alpha=70^{\circ}$ solutions, because in this case there is almost no sensitivity to variations in $\alpha$. The response to varying $\alpha$ is greatest in the range $500 \leq T \leq 1000 \mathrm{MeV}$ and diminishes rapidly below $T=100 \mathrm{MeV}$.

This figure may also be used to illustrate qualitatively the effect of positrons on the observed intensity. In the period from 1970 to 1980 , the full lines should be interpreted as modulated

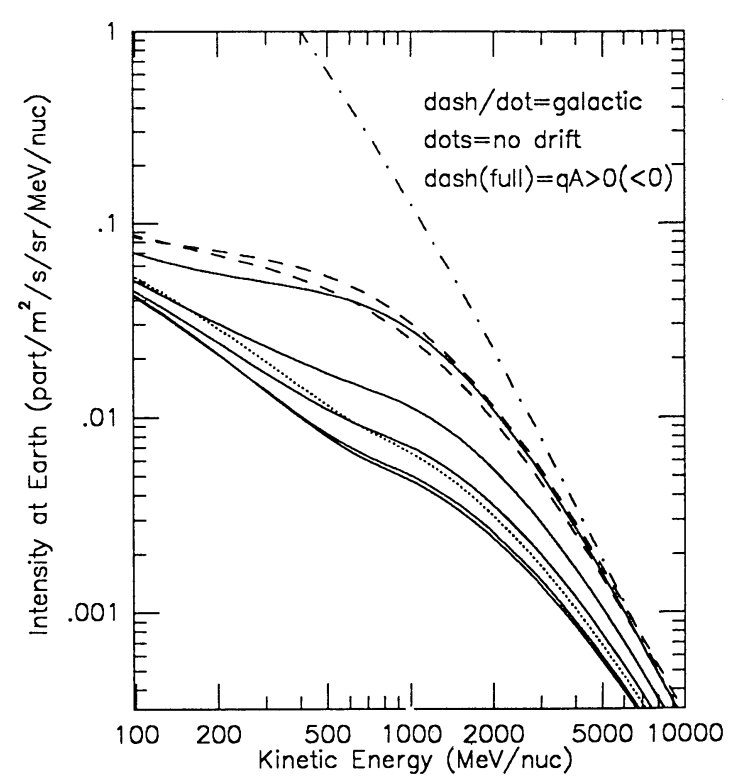

FIG. 2.-Interstellar (dot-dashed line) and calculated modulated electron spectra at $1 \mathrm{AU}$. Full lines from top to bottom are for neutral sheet tilt angles $\alpha=0^{\circ}, 10^{\circ}, 20^{\circ}, 40^{\circ}$, and $70^{\circ}$ and represent negatrons in $A>0$ cycle $(1970$ 1980). Two dashed lines are for $\alpha=0^{\circ}$ and $70^{\circ}$ and represent positrons in the same cycle. Dotted curve shows a no-drift solution for comparison.

negatron spectra, which respond strongly to sheet waviness, while the dashed solutions are for positrons. (This assumes identical shapes for the Galactic positron and negatron spectra, varying only their relative normalization.) Therefore, in such a cycle, a small interstellar positron fraction of, say $f_{+}=0.1$ to 0.2 , may be boosted by a very large amount in the modulated intensities because the negatron intensities drop by an order of magnitude in such a cycle. By the same argument, the modulated positron fraction will be suppressed by a large amount in adjacent $(A<0)$ magnetic cycles.

These points are quantitatively illustrated in Figure 3. Figure $3 a$ shows the response of negatrons to varying $\alpha$ for both signs of $A$. For Figure $3 b$ a (reasonable) positron fraction, $f_{+}=0.2$, is assumed, and we emphasize that the loop for positrons is traversed in the opposite direction than the one for negatrons. In Figure $3 c$ these intensities are added, showing clearly that the total electron response to sheet waviness is much reduced below that for negatrons only. Finally, Figure $3 d$ shows that when $A<0$, the positron fraction at $1 \mathrm{GeV}$ may fall as low as $f_{+} \approx 0.05$, while, when $A>0, f_{+}$may become greater than 0.5 , so that positrons actually dominate the modulated total electron intensity. We lament the fact that there are at present insufficient observations to confirm or deny these predictions.

The loops in Figure 3 show only the response to changing $\alpha$ as a modulation agent. It must be clearly borne in mind that the loops will be modified by variations of other modulation parameters with solar activity. Examples of these are increased scattering toward solar maximum conditions, leading to lower diffusion coefficients, and an increase of the effective scalar magnitude of $B$, as observed by Winterhalter, Smith, and Slavin (1988). These effects would reduce the width of the loops and, in particular, would strongly modify the abrupt "jump" at $\alpha=70^{\circ}$ to a more gradual transition.

As stated, these solutions are for the most realistic modula- 

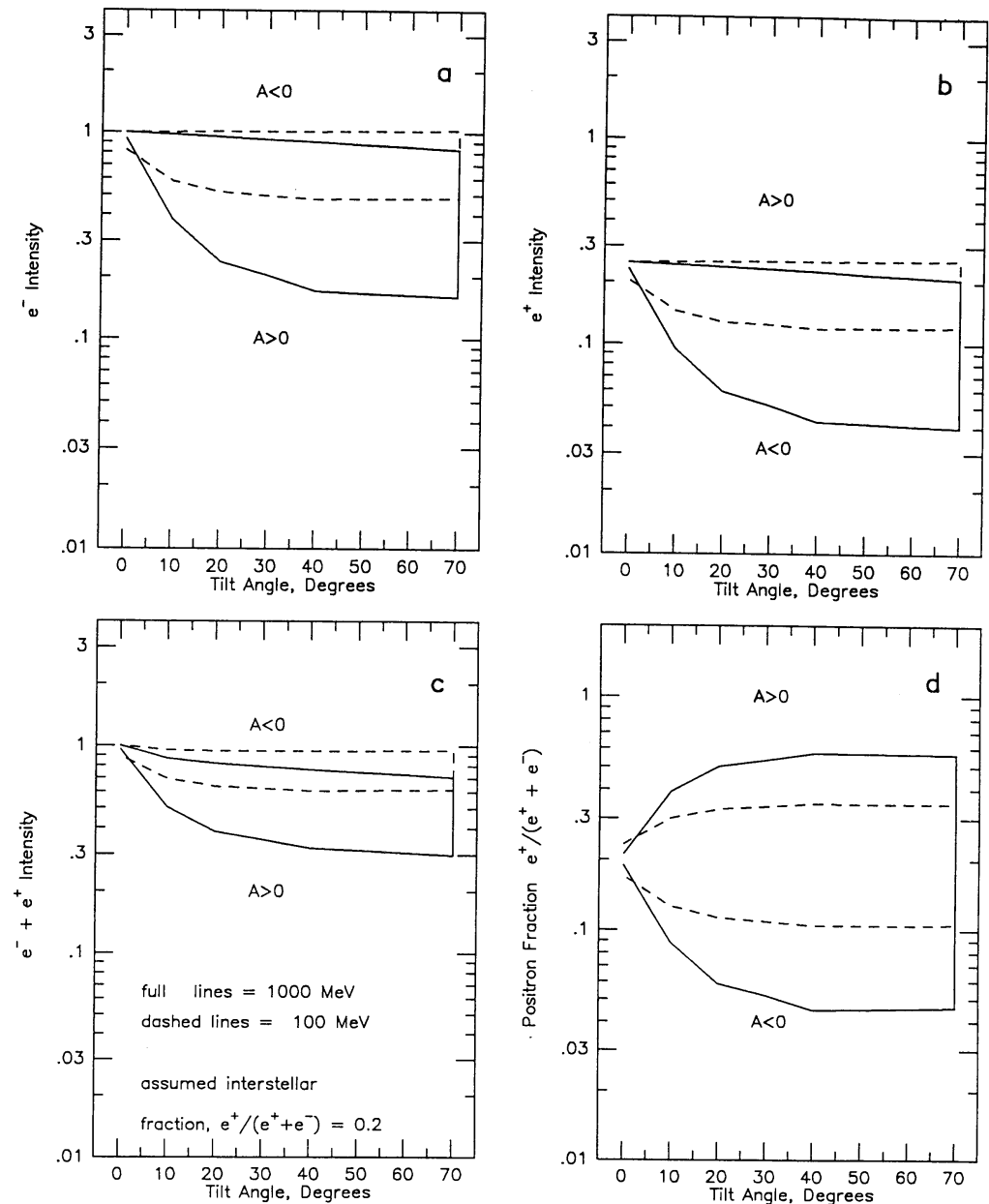

Fig. 3.-Full and dashed lines show $1000 \mathrm{MeV}$ and $100 \mathrm{MeV}$ intensity excursions as function of sheet tilt angle, respectively; (a) for negatrons; (b) for positrons, assuming an interstellar fraction $f_{+}=20 \% ;(c)$ combined $e^{+}+e^{-} ;(d)$ modulated fraction.

tion parameters available. We have, however, verified that a wide range of variations of these parameters always produce similar loop structures. The influence of varying the positron content can easily be calculated from Figures $3 a$ and $3 b$. We quote, for example, that for an assumed interstellar $f_{+}=0.0$, $0.1,0.2,0.3,0.4$, and 0.5 , the "jump" at $\alpha=70^{\circ}$ for $1 \mathrm{GeV}$ electrons in Figure $3 c$ reduces from 5.0 to $3.0,2.2,1.7,1.25$, and 1.0. Similarly, the jump in the modulated positron fraction of Figure $3 d$ has the values $17,13,9,7$, and 5.5 for an intestellar $f_{+}=0.1,0.2,0.3,0.4$, and 0.5 .

\section{COMPARISON WITH ELECTRON OBSERVATIONS}

Measured positron fractions below about $5 \mathrm{GeV}$, as shown in Figure 1, can be corrected to their interstellar values from a knowledge of the neutral sheet tilt angles at the time of observation using the above model calculation. Those of Fanselow et al. (1969) were made during the solar minimum period of 1965 and shortly thereafter in 1966, when $A<0$. Figure $3 d$ suggests that for periods with low neutral sheet tilt (such as in 1965), the measured positron fraction should be well representative of interstellar values, but that, in 1966 , the positron fraction ought to be suppressed somewhat by the modulation. The measured tilt angles of Hoeksema, Wilcox, and Scherrer (1983) only go back to 1977 , however, and this makes the required amount of upward correction to these 1965 and 1966 points unknown. We therefore leave them uncorrected.

The measurements of Daugherty, Hartman, and Schmidt (1975) were made in 1972, and those of Hartman and Pellerin (1976) in 1974. Periods on both sides of an $A>0$ solar minimum, such as from 1972 to 1974 and from 1977 to 1979 will potentially have a strongly enhanced positron content in the electron intensities. During these periods, the tilt angles (as inferred from the Hoeksema data for 1977 to 1979) should typically be in the range of $30^{\circ}$ to $50^{\circ}$. Figure $3 d$ suggests that the modulated positron fraction can easily be double the interstellar value during such times. As a first step, and in the absence of more detailed field measurements, we therefore lowered these latter ratios in Figure 1 by a factor of 2, with the corrected results shown in Figure 4. The net effect of these corrections is to reduce the discrepancy between the calculated values of Protheroe (1982) and the measurements greatly. This lends credence to the role of a wavy neutral sheet effects in the modulation.

When electron intensities at $600<T<1000 \mathrm{MeV}$ are plotted against tilt angle from 1977 to 1985 (van Staden 1989) they show a loop of about the same size as in Figure $3 c$, but this loop is traversed in the opposite direction, that is, in the same sense as for positively charged nuclei. This suggests that 


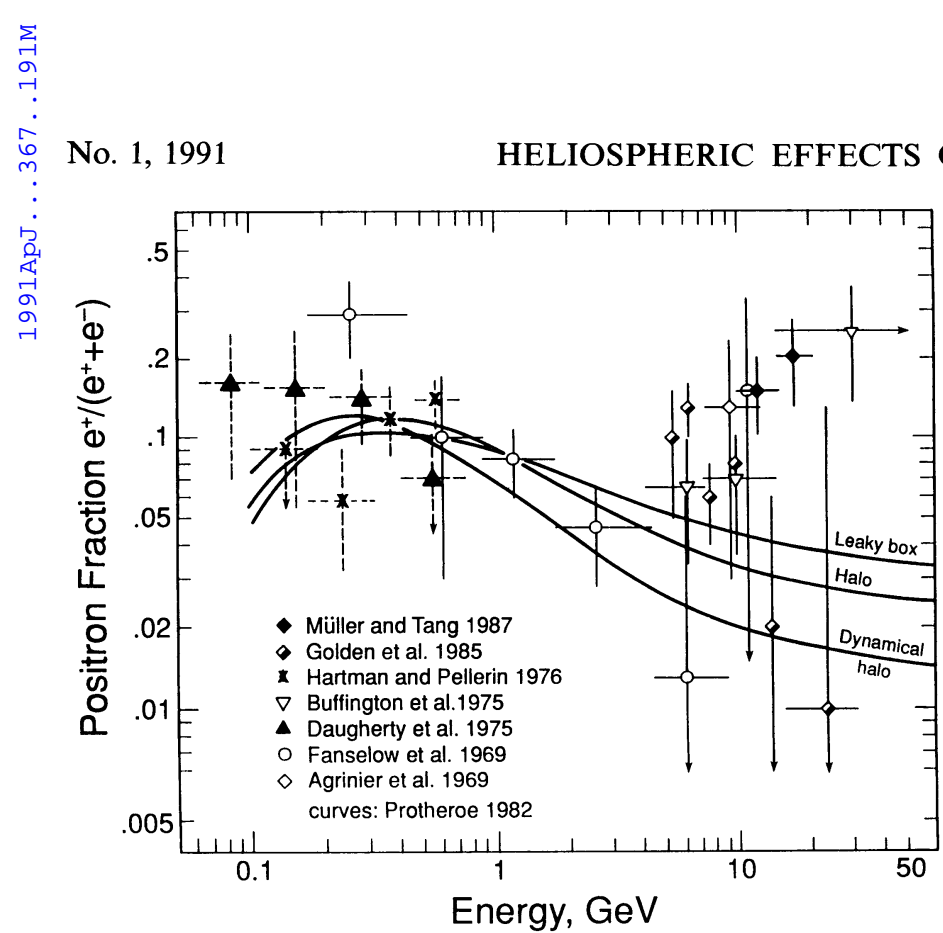

FIG. 4.-Same as Fig. 1, with points with dashed error bars demodulated according to the best present estimate. Key to references: $\diamond$, Müller and Tang (1987); $\diamond$, Golden et al. (1985); *, Hartman and Pellerin 1976; $\nabla$, Buffington et al. (1975); $\boldsymbol{\Delta}$, Daugherty et al. (1985); $\bigcirc$, Fanselow et al. (1969); $\diamond$, Agrinier et al. (1969); curves; Protheroe (1982).

the positron correction (from Figures $3 a$ and $3 b$ to Figure $3 c$ ) is not sufficient to explain the time variations of electrons in detail over a solar cycle. Possible reasons for this are (1) variation of diffusion coefficients and drift velocity with solar activity, (2) time-dependent effects in the modulation that may be the primary origin of hysteresis effects, (3) a possible contribution of Jovian electrons, which are clearly observed below 100 $\mathrm{MeV}$, and (4) their possible acceleration in the solar wind termination shock. Such acceleration effects are discussed in $\S$ VII of this paper.

\section{THE HELIUM TO ELECTRON RATIO}

Garcia-Munoz et al. $(1986,1987)$ have most explicitly addressed the relative modulation of electrons and nuclei. They considered the intensity ratio of 70-95 MeV per nucleon helium to that of $600-1000 \mathrm{MeV}$ electrons (see Fig. 5). The particles were chosen because they have comparable magnetic rigidities. The ratio clearly increased during the 1969 to 1971 polarity reversal and fell again during the 1980 reversal. The authors pointed out that these asymmetric transitions during subsequent solar maxima clearly indicate charge-dependent modulation effects as predicted by drift models. Modeling the helium/negatron ratio, using a standard Parker spiral field with a magnitude $B_{e}=5 \mathrm{nT}$ and without the solar polar field modification of Jokipii and Kóta (1989) wavy neutral sheet drift models, such as those used in Figures 2 and 3, predicts that this ratio should change by a factor of at least 70 , more than 10 times larger than the observed change (Potgieter and Burger 1990).

However, if a realistic field model is used in the calculation and when the positron content in the electron flux is taken into proper account, the model of the previous section predicts a much more reasonable value for these transitions. First, note that a plot of the helium/electron ratio is strikingly similar to that of the positron fraction, $f_{+}$, in Figure $3 d$. Differences in rest mass, interstellar input spectra, energy loses, etc., have almost no influence on these ratios. We therefore show only the "jump" at $70^{\circ}$ tilt, corresponding to the above mentioned, measured, transitions during polarity reversals.

Figure 6 demonstrates that for $B_{e}=5 \mathrm{nT}$ this transition decreases from a factor of 40 to 12 when the interstellar positron fraction increases from 0 to 0.3 . Doubling the value of $B_{e}$ to $10 \mathrm{nT}$ (halving the drift velocities everywhere) and taking a positron fraction of 0.3 can make this transition as low as a factor 5. The most realistic values are probably in the range $8<B_{e}<10 \mathrm{nT}$ and $0.1<f_{+}<0.2$, giving a transition in the range 6 to 12 , which we consider in reasonable agreement with observations.

Bearing in mind that other possibilities must also be addressed, we conclude that the similarity in the shapes of the electron and helium intensities during the 1975 sunspot minimum can be understood, directly, if the contribution of positrons to the electron flux becomes so large that the expected peak behavior for negatrons is diminished. In addition, it can be inferred from the measurements of Christian (1989) that anomalous He contributes up to $\approx 15 \%$ of the 70-95 MeV per nuclear helium flux seen at solar minimum. This makes the He time profile somewhat more peaked than expected for fully stripped $\mathrm{He}$. In addition, further support for these predictions is found in the recently reported, preliminary measurements of Garcia-Munoz et al (1990), which indicate that during the 1987 cosmic-ray maximum the electron intensity was very sharply peaked relative to the He intensity time

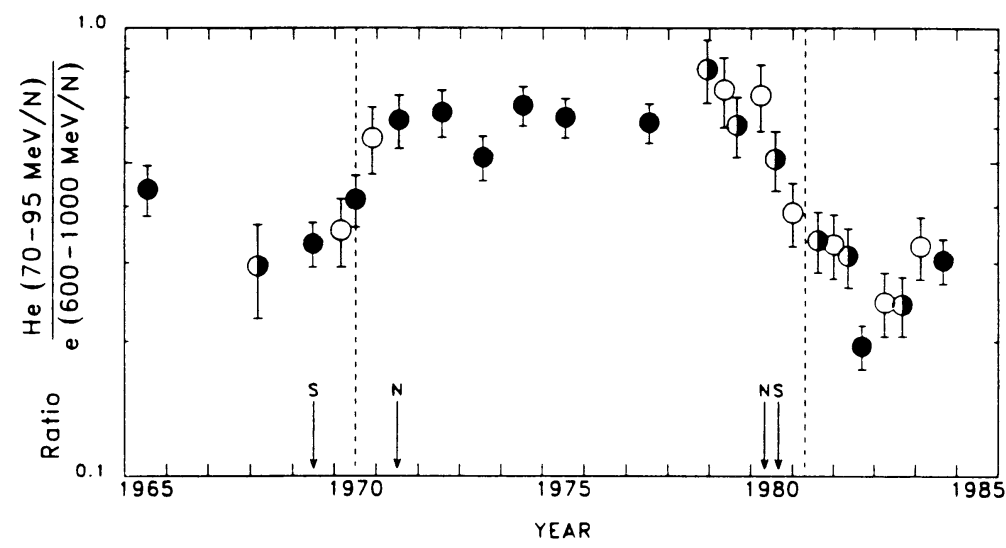

FIG. 5.-Measured helium/electron ratio at Earth, reproduced from Garcia-Munoz et al. (1987) 


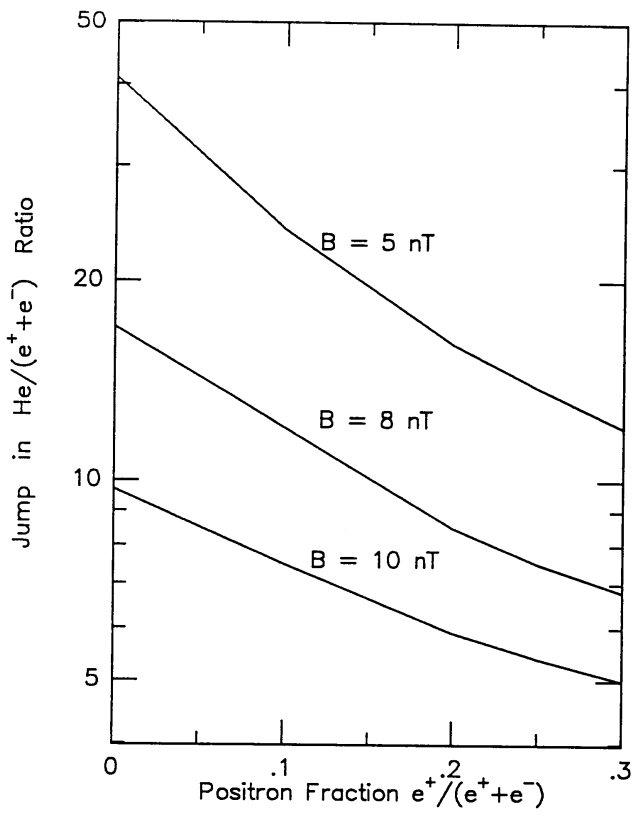

Fig. 6.-Calculated jump in the helium to electron ratio as function of $f_{+}$to be compared with the transition at solar maximum shown in Fig. 5. The $B$-values refer to effective magnetic field strengths, $B_{e}$, at Earth.

profile. This is expected from our model because during this maximum the positron flux is strongly suppressed. These qualitative statements require a more quantitative proof in terms of more elaborate models.

\section{SECONDARY ELECTRONS FROM SOLAR SYSTEM OBJECTS}

The previous sections demonstrate that realistic field models and a small but appreciable positron content significantly modify the electron modulation. In this and the next section we address the sources of cosmic-ray electrons. If these electrons are not of interstellar origin - as is commonly perceived - this may further alter their modulation and time-intensity profiles as observed in the inner heliosphere.

A possible alternative source of electrons in the solar system is the production of secondary electrons by cosmic-ray protons striking solar system objects. The source strength depends in general on the details of the specfic target. However, a crude estimate may be obtained by assuming that the source per unit area is that observed above the Earth's atmosphere. A variety of observations of albedo electrons were reviewed by Daniel and Stephens (1974) and reproduced in their Figure 8. From this we take a fiducial value of the differential spectrum of albedo electrons at $100 \mathrm{MeV}$ of $j(100)=10^{-4} \mathrm{~cm}^{-2} \mathrm{~s}^{-1}$ $\mathrm{MeV}^{-1} \mathrm{sr}^{-1}$. The upper bound (assuming that all particles escape) of the source strength for these particles at the Earth is then $Q_{E}=3 \times 10^{15} \mathrm{MeV}^{-1} \mathrm{~s}^{-1}$. In reality, the source strength is probably $10 \%$ of this because of the Earth's magnetic field. The source strengths of other objects may be similarly calculated by scaling by the area.

For a given source $Q(T)$, assuming isotropic diffusion, we can compute the isotropic intensity from diffusion theory as

$$
j(T, r)=\frac{Q(T) w}{16 \pi^{2} r \kappa},
$$

where $w$ is the particle speed and the diffusion coefficient $\kappa$ is a constant for a given $T$. This gives the intensity just outside the
Earth at $100 \mathrm{MeV}$ as $4 \times 10^{-7} \mathrm{~cm}^{-2} \mathrm{~s}^{-1} \mathrm{MeV}^{-1} \mathrm{sr}^{-1}$, a factor of 20 below the observed electron flux. This could be increased by a factor of 10 or so if the diffusion is highly anisotropic along the magnetic field. This value is high enough to contribute to the observed flux. It does, however, fall off with distance from the source as $1 / r$.

A similar calculation gives the source strength at Jupiter as $4 \times 10^{17} \mathrm{MeV}^{-1} \mathrm{~s}^{-1}$. Chenette (1980) calculated that the total magnetospheric contribution of Jovian electrons below about $20 \mathrm{MeV}$ is $3 \times 10^{25} \mathrm{~s}^{-1}$. The secondary contribution is, therefore, quite negligible. This cannot affect the intensity at Earth.

Finally, consider the possible effects of numerous cometesimals in the inner solar system, as has been proposed by some (e.g., Frank 1985). If there are $N_{c}$ such objects inside 1 A.U., each having an area $A_{c}$, then, if they are several interaction lengths $\left(\approx\right.$ several $\left.\mathrm{g} \mathrm{cm}^{-2}\right)$ or more thick, then the total source within 1 A.U. is

$$
Q_{c} \approx N_{c} A_{c} \frac{Q_{E}}{4 \pi R_{E}^{2}}
$$

and the resulting intensity at 1 A.U. is, again using simple diffusion theory, from equation (5)

$$
j_{c} \approx \frac{Q_{c} w}{16 \pi^{2} r \kappa} .
$$

If we use the numbers from Frank, Sigworth and Craven (1985), giving $N_{c}=10^{14}$ and $A_{c}=10^{6}$, we get $j_{c}=4 \times 10^{-9}$ for the flux of $100 \mathrm{MeV}$ electrons at $1 \mathrm{~A} . \mathrm{U}$. This is again much too small to be important.

We have made similar calculations for the moons and other planets, interplanetary dust, and asteroids. Their contributions also seem to be very small.

Our conclusion is that the contribution of secondary electrons from solar system objects, except the Earth, is clearly too small to be of importance. The contribution of the Earth to observations in near Earth space is less clear and probably merits a more accurate computation.

\section{ACCELERATION AT THE SOLAR WIND TERMINATION SHOCK: ANOMALOUS ELECTRONS}

We now consider the possibility that significant numbers of electrons are accelerated at the termination shock of the solar wind, which is thought to be the origin of the "anomalous" cosmic rays observed in the inner solar system. This anomalous component has been the subject of considerable discussion and speculation since its discovery in the early 1970s. It consists of enhanced fluxes of helium, nitrogen, oxygen, neon, and recently protons, carbon, and argon in a region of the energy spectrum ranging from a kinetic energy of $20 \mathrm{MeV}$ to perhaps $300 \mathrm{MeV}$.

Pesses, Jokipii, and Eichler (1981) pointed out that many features of the anomalous component could be explained if the acceleration of freshly ionized interstellar particles, proposed by Fisk, Kozlovsky, and Ramaty (1974), occurs by the mechanism of diffusive shock acceleration at the termination shock of the solar wind.

Jokipii (1986) presented results from a more detailed twodimensional numerical simulation of this model, in which the full transport equation (including drift, diffusion, convection, and energy change) was solved. In this model, it was clear that (a) particles could be accelerated very efficiently at the termination shock of the solar wind and $(b)$ the essential features of the 
spectrum and the gradients could be explained very naturally in terms of this model, with reasonable values for the parameters. The same model heliosphere also successfully accounts for many properties of the modulation of Galactic cosmic rays, as discussed above. At present this appears to be the consensus model for the origin of the anomalous component. Here we report the results of applying this numerical model to the acceleration of electrons. The numerical model follows the acceleration of low-energy particles injected into the solar wind and then accelerated at the termination shock. Hence, the source function $Q$ is set to correspond to the injection of low-energy electrons at the termination shock, although the results would be similar for injection at any point interior to the shock. The method of solution is then to follow these particles in time as they are accelerated at the shock and propagate throughout the heliosphere. The model is two-dimensional, which forces a flat current sheet. The diffusion coefficient used in these calculations differs from that used in the modulation calculations of $\$ \S$ II-IV. We take

$$
\kappa_{\|}=\kappa_{0} P^{1 / 2} \beta\left|B_{e} / B\right|,
$$

where $\kappa_{0}$ is $5 \times 10^{21} \mathrm{~cm}^{2} \mathrm{~s}^{-1}$ for $P$ in GV. The ratio of perpendicular to parallel diffusion is given by

$$
\frac{\kappa_{\perp}}{\kappa_{\|}}=0.03
$$

and $\kappa_{\mathrm{T}}$ is as in $\S$ III. The characteristic time for these processes to approach a steady state is found to be 2-3 yr.

Figure 7 shows the energy spectrum of the accelerated electrons near the heliographic equator, both at the shock (at a radius of $80 \mathrm{~A} . \mathrm{U}$.), and at a heliocentric radius of $10 \mathrm{~A} . \mathrm{U}$., for the case where particles are injected at some low energy at the shock. An absorbing boundary was placed at 110 A.U. to represent loss to the Galaxy. This is presented to illustrate that, given a source of "injected" electrons at some low momentum, electrons are accelerated efficiently at the shock, as are ions. It

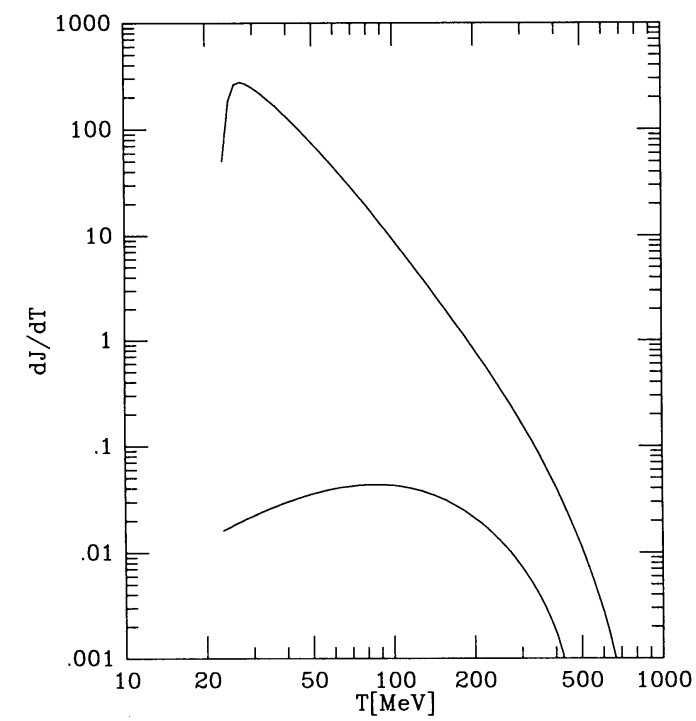

Fig. 7.-Computed energy spectra of anomalous electrons in a twodimensional simulation for the parameters given in the text. Upper curve is the spectrum at the shock (80 A.U.), and lower curve is the spectrum at $10 \mathrm{~A} . \mathrm{U}$. Both curves were computed for latitudes near the equatorial plane. Injection was at $1.3 \mathrm{MeV}$. Units of $d j / d T$ are arbitrary. is clear that electrons with energies of $100 \mathrm{MeV}$ or higher may readily be produced.

A major problem in the diffusive shock acceleration of cosmic rays is to understand the process by which particles become injected into the acceleration process. The standard theory, which explains a number of diverse phenomena, applies strictly only to charged particles with speeds significantly above the background plasma flow speed $U$. Hence, most thermal particles are excluded. The success of the anomalous component model in explaining the composition lies in the expectation that recently ionized interstellar ions have a high speed relative to the local plasma thermal speeds. The composition reflects that of the pickup ions and not that of the thermal gas. Beyond this, the injection problem remains to be understood in terms of first principles.

The question of diffusive acceleration of electrons has been studied even less. Electrons clearly have a high thermal speed, relative to the background plasma flow and, for that reason, may be better candidates for acceleration than the much cooler ions. But, also, because of their small mass, any electric fields at the shock will affect them more than ions.

Assume for a simple order-of-magnitude argument that there are 5 (A.U. $/ r)^{2}$ electrons $\mathrm{cm}^{-3}$ in the solar wind. For a shock at 50 A.U., these electrons have a flux of $8 \times 10^{4} \mathrm{~cm}^{-2}$ $\mathrm{s}^{-1}$. Consideration of acceleration at a strong parallel shock shows that less than $1 \%$ of these electrons need to be successfully injected in order to account for the entire cosmic-ray electron intensity observed at a few hundred MeV. This efficiency decreases as the shock compression ratio decreases, and shocks with compression ratios less than 3 cannot produce the required flux. It is therefore possible that such locally accelerated electrons may alter the electron flux observed at $\mathrm{MeV}$ energies.

We wish to point out, in addition, that there is a wellestablished source of superthermal (and, in fact, quite energetic) electrons in the inner solar system. Energetic electrons up to some $20 \mathrm{MeV}$ energy, emitted by the Jovian magnetosphere, dominate the quiet-time electron spectrum. Indeed, the entire near Earth spectrum below the turnup at about $20 \mathrm{MeV}$ in the spectrum (see, e.g., Fig. 18 in Meyer 1969) is probably of Jovian origin (Eraker 1982). These electrons will reach the termination shock, and there they will certainly be accelerated because, already having high energies, they do not suffer the "injection uncertainty" of thermal solar wind electrons.

We have modeled this situation, precisely as in the anomalous component models discussed above, except that the "injected" particles are now taken to be a spectrum injected at the orbit of Jupiter, corresponding to the electrons leaking from the Jovian magnetosphere. Since the model available at present contains only two spatial dimensions (heliocentric radius $r$ and polar angle $\theta$ ), the source at Jupiter was taken to be an equatorial ring at 5 A.U. and to be a power law in energy $Q \propto T^{-4.8}$, with a sharp cutoff above some $30 \mathrm{MeV}$. We find that the resulting spectrum at Earth below some $20 \mathrm{MeV}$ is close to the observed power law $j(T) \propto T^{-2}$. The source strength required to produce the observed flux below $20 \mathrm{MeV}$ is quite close to the $3 \times 10^{25} \mathrm{~s}^{-1}$ calculated by Chenette (1980). In fact, our model may be regarded as extending his model to consider transport of the electrons in a full model heliosphere, including acceleration at the termination shock.

At higher energies $(\approx 100 \mathrm{MeV})$ in the inner heliosphere, we find evidence of an enhancement in the computed spectrum, 


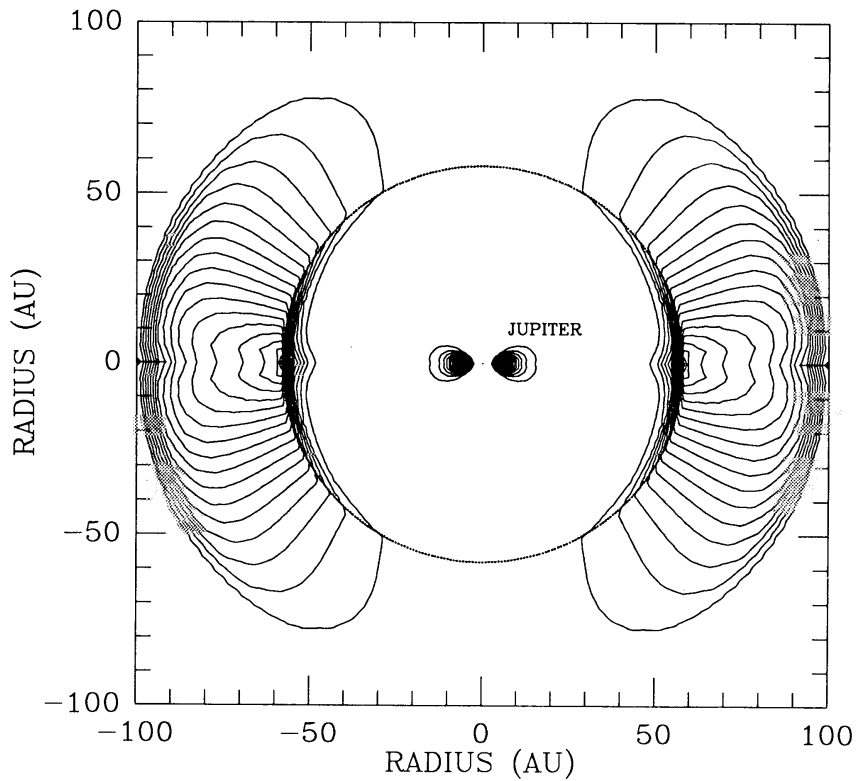

FIG. 8.- Contours of equal intensity in a meridional plane, for electrons of $\simeq 10 \mathrm{MeV}$ energy escaping from the Jovian magnetosphere. Clearly seen is a maximum near the shock (indicated by dotted circle at 60 A.U.), showing the acceleration there. The model has an absorbing boundary at 100 A.U. Nominal solar wind parameters were used, and the transport coefficients were taken to be those used in Fig. 7 .

which are Jovian electrons which have propagated out to the termination shock, have been accelerated there, and have then returned to the inner solar system. These would then be called the anomalous electrons. To demonstrate that electrons are indeed accelerated at the shock, we show in Figure 8 a contour plot of the intensity in a meridional plane, for a moderate energy of $10 \mathrm{MeV}$, below the cutoff in Jovian injection. The peak in intensity due to injection at the orbit of Jupiter is clearly seen at 5 A.U. There is, in addition, a second maximum at the shock, which corresponds to lower energy Jovian electrons which have been convected to the shock and then accelerated there. These reaccelerated Jovian electrons extend to energies $\simeq 200 \mathrm{MeV}$ and higher.

We conclude that acceleration of Jovian electrons at the solar wind termination shock occurs and that it may significantly affect the observed time history of the electron flux, especially because the acceleration may be most efficient during solar maximum periods when the termination shock may be stronger (or when there may be more traveling interplanetary shocks in the outer heliosphere). The question of whether the enhancements are observable in the inner heliosphere depends on the transport parameters.
We also suggest that observations in the outer heliosphere near the termination shock may well show evidence for anomalous electrons at energies of the order of $200 \mathrm{MeV}$. Because of differences in the relative importance of adiabatic cooling for nuclei and electrons, radial density gradients for electrons should be much higher than for nuclei. For this reason one expects high electron intensities near the termination shock.

\section{SUMMARY AND CONCLUSIONS}

Drift models of the cosmic-ray modulation cycle are quite successful in describing the modulation of cosmic-ray nuclei. It was shown here that when proper account is taken of the modifying role of positrons and when realistic heliospheric field models are used, the long-standing problem of electron modulation is also solved to a large extent. The realistic field models include both the solar field modification of Jokipii and Kóta (1989) and the moderated drift effects due to longwavelength turbulence in the entire heliosphere, as pointed out by Burger and Moraal (1990). Detailed model applications are now needed to resolve this question further. We also wish to point out that there may be a real possibility of detecting electrons accelerated at the termination shock on the Pioneer/ Voyager missions. If cosmic-ray electrons of kinetic energies $\lesssim 100 \mathrm{MeV}$ are not of true interstellar origin, standard modulation theories do not apply to their heliospheric propagation and modulation. Our preliminary estimates indicate that the production of secondary electrons in solar system objects is probably too low to account for any significant fraction of cosmic-ray electrons. We do find, however, that negatrons, emitted by the magnetosphere of Jupiter and accelerated by the solar wind termination shock, provide an attractive source of negatrons up to several hundred $\mathrm{MeV}$. Their modulation has to be studied in much greater detail.

In conclusion, detailed new electron experiments which can provide accurate spectra, their temporaral variations, a definite separation of positrons and negatrons, as well as simultaneous measurements of the density gradients of these two electron species, may provide the most important new information to unravel the modulation process. In addition, it is clear from the analysis that the modulation of electrons must be understood in order to interpret measurements of the positron fraction of $\lesssim \mathrm{GeV}$ electrons properly.

The work of R. A. M. and H. M. was supported in part by NASA under contracts NAS-7-918, NAS 5-727, and grant NGR-05-002-160. J. R. J. was supported, in part, by the National Science Foundation under grant ATM-8618260 and by the National Aeronautics and Space Administration under grant NAGW-1931.

\section{REFERENCES}

Agrinier, B., et al. 1969, Lettere Nuovo Cimento, B1, 53.

Buffington, A., Orth, C. D., and Smoot, G. F. 1975, Ap. J., 199, 699.

Burger, R. A., and Moraal, H. 1990, Proc. 21st Internat. Cosmic Ray Conf. (Adelaide), 5, 272.

Chenette, D. 1980, J. Geophys. Res., 85, 2243.

Christian, E. C. 1989, Ph.D. thesis, California Institute of Technology.

Cummings, A. C., Mewaldt, R. A., Stone, E. C., and Webber, W. R. 1990, Proc. 21st Internat. Cosmic Ray Conf. (Adelaide), 6, 206.

Cummings, A. C., Stone, E. C., and Webber, W. R. 1990, Proc. 21st Internat. Cosmic Ray Conf. (Adelaide), 6, 190.

Daniel, R. R., and Stephens, S. A. 1974, Rev. Geophys. Space Phys., 12, 233.

Daugherty, J. K., Hartman, R. C., and Schmidt, P. J. 1975, Ap. J., 198, 493.

Eraker, J. H. 1982, Ap. J., 257, 862.
Fanselow, J. L., Hartman, R. C., Hildebrand, R. H., and Meyer, P. 1969, Ap. J., 158, 771.

Fisk, L. A., Kozlovsky, B., and Ramaty, R. 1974, Ap. J. (Letters), 190, L35.

Frank, L. A., Sigworth, S. B., and Craven, J. D. 1985, Geophys. Res. Letters, 13 307.

Garcia-Munoz, M., Meyer, P., Pyle, K. R., Simpson, J. A., and Evenson, P. 1986, J. Geophys. Res., 91, 2858

. 1987, Proc. 20th Internat. Cosmic Ray Conf. (Moscow), 3, 303.

Garcia-Munoz, M., Meyer, P., Pyle, K. R., Simpson, J. A., Evenson, P., and Tuska, E. 1990, Proc. 21st Internat. Cosmic Ray Conf. (Adelaide), 6, 69.

Golden, R. L., Mauger, B. G., Horan, S., Badwhar, G. D., Lacy, J. L., Zipse, J. E., Daniel, R. R., and Stephens, S. A. 1985, Proc. 19th Internat. Cosmic Ray Conf. (La Jolla), 2, 374. 
Hartman, R. C., and Pellerin, L. J. 1976, Ap. J., 204, 927.

Hoeksema, J. T., Wilcox, J. M., and Scherrer, P. H. 1983, J. Geophys. Res., 88 , 9910.

Jokipii, J. R. 1986, J. Geophys. Res., 91, 2929.

II Jokipii, J. R., and Kopriva, D. A. 1979, Ap. J., 234, 384

-I Jokipii, J. R., and Kóta, J. 1989, Geophys. Res. Letters, 16, 1.

Jokipii, J. R., and Thomas, B. 1981, Ap. J., 243, 1115 .

Kóta, J., and Jokipii, J. R. 1983, Ap. J., 265, 573.

McKibben, R. B. 1989, J. Geophys. Res., 94, 17021

Meyer, P. 1969, Ann. Rev. Astr. Ap., 7, 1

Moraal, H. 1990a, Proc. 21st Internat. Cosmic Ray Conf. (Adelaide), 6, 140.
Moraal, H. 1990b, Proc. 21st Internat. Cosmic Ray Conf. (Adelaide), 6, 194 Mueller, D., and Tang, K.-K. 1987, Ap. J., 312, 183.

Parker, E. N. 1965, Planet. Space Sci., 13, 9.

Pesses, M. E., Jokipii, J. R., and Eichler, D. 1981, Ap. J. (Letters), 246, L85.

Potgieter, M. S., and Burger, R. A. 1990, Astr. Ap., in press.

Protheroe, R. J. 1982, Ap. J., 254, 391.

van Staden, M. L. 1989, M.S.thesis, Potchefstroom University, South Africa.

Winterhalter, D., Smith, E. J., and Slavin, J. A. 1988, Proc. 6th Solar Wind

Conf., ed. V. J. Pizzo, P. E., Holzer, and D. G. Sime (Boulder: NCAR Technical Note NCAR/TN-306), p. 587.

J. R. JoKIPII: Department of Planetary Sciences, Lunar and Planetary Laboratory, University of Arizona, Tucson, AZ 85721

R. A. MewaldT, and H. MoraAl: 220-47 Downs Laboratory, California Institute of Technology, Pasadena, CA 91125 